Article

\title{
Passivity-Based Nonlinear Excitation Control of Power Systems with Structure Matrix Reassignment
}

\author{
Jianyong $\mathrm{Li}^{1}$, Yanhong Liu ${ }^{2, *}$, Chunwen $\mathrm{Li}^{3}$ and Bing Chu ${ }^{4}$ \\ ${ }^{1}$ School of Computer Science, Zhengzhou University of Light Industry, Zhengzhou 450002, China; \\ E-Mail: lijianyong@zzuli.edu.cn \\ ${ }^{2}$ School of Electrical Engineering, Zhengzhou University, Zhengzhou 450001, China \\ ${ }^{3}$ Department of Automation, Tsinghua University, Beijing 100084, China; \\ E-Mail: lcw@mail.thu.edu.cn \\ ${ }^{4}$ Department of Electronics \& Computer Science, University of Southampton, Southampton SO17 1BJ, \\ UK; E-Mail: b.chu@ecs.soton.ac.uk \\ * Author to whom correspondence should be addressed; E-Mail: liuyh@zzu.edu.cn; \\ Tel.: +86-371-67781412; Fax: +86-371-67783113.
}

Received: 10 April 2013; in revised form: 12 August 2013 / Accepted: 12 August 2013 /

Published: 20 August 2013

\begin{abstract}
Passivity-based control is widely used in electronic circuit systems because it can utilize their internal structures to facilitate the controller design. In this paper, we first propose a dissipative Hamiltonian realization of power systems and discuss the disadvantages of the traditional passivity-based excitation controller. Then, a novel excitation controller is put forward to reassign the interconnection and dissipative matrix, and the corresponding Hamiltonian function. Simulation results verify that the proposed controller can effectively improve the transient stability of the power system.
\end{abstract}

Keywords: hamiltonian realization; interconnection and damping assignment; passivity-based control; power systems; stabilization

\section{Introduction}

The port-controlled Hamiltonian $(\mathrm{PCH})$ system has gained increasing interest in the control and energy community [1]. The main characteristic of the $\mathrm{PCH}$ system is that the structural 
information is available in the model description: the total energy of the system is represented by the Hamiltonian function, which can be usually used as a Lyapunov function in stability analysis, while the interconnection property is explicitly described by the interconnection and damping matrix.

By utilizing the energy concept and the internal structural property, several methods have been developed for the controller design of PCH systems [2-5]. Interconnection and damping assignment passivity-based control (IDA-PBC) is a widely used state-feedback technique which can stabilize the considered nonlinear systems by shaping the energy and re-constructing the structure matrices. So far, IDA-PBC has been successfully applied to electromechanical systems [6,7], power electronics [8], and power systems [9-15].

For power systems, the energy shaping is generally achieved by modifying the energy transfer pattern between the mechanical and the electrical components, which is obtained by injecting damping into the electrical dynamic. The conventional IDA-PBC excitation controller cannot directly reassign the mechanical damping in the swing equation, though the damping in the swing equation is very important for transient stability improvement. In this paper, we propose a novel IDA-PBC excitation controller to enhance the transient stability of power systems by choosing a convenient interconnection matrix and find a solution for the matched partial derivative equation. The proposed excitation controller not only provides a compensation damping into electrical dynamic equation, but also into the mechanical swing equation. Simulation results verify that, compared to the conventional energy-shaping method [14] and PSS+AVR controller [16], the proposed control scheme can effectively improve the transient stability of the power system.

The rest of the paper is organized as follows. In section 2 we provide some preliminary results on the IDA-PBC method. In Section 3 we propose the dynamic model of the single machine infinite bus power systems and put forward a novel IDA-PBC excitation controller. In Section 4, we simulate the power system, to illustrate the effectiveness of the proposed control scheme. Section 5 summarizes the results and concludes.

\section{IDA-PBC Methodology of Nonlinear Systems}

Consider the following nonlinear system:

$$
\dot{x}=f(x)+g(x) u
$$

where $x \in R^{n}$ is the state vector and $u \in R^{m}$ is the control input. $f(x)$ and $g(x)$ are smooth vector functions in the appropriate dimensions.

Suppose the system can be represented as the following PCH formulation,

$$
\dot{x}=[J(x)-R(x)] \nabla H(x)+g(x) u
$$

where $J(x)=-J^{T}(x)$ and $R(x)=R^{T}(x) \geq 0$ are the interconnection and damping matrices respectively. $H(x)$ is the Hamiltonian function, which represents the total energy stored in the system. $\nabla H(x)$ is the gradient vector function of $H(x)$ with respect to $x$.

If the Hamiltonian function $H(x)$ has a strict minimum at the equilibrium point, it can be chosen as the Lyapunov candidate for the stability analysis and passivity-based controller design. Unfortunately, for a 
physical system the Hamiltonian function does not always have a minimum at the desired equilibrium or the dynamic performance of the system is not satisfactory; so the IDA-PBC is always needed to shape the energy function, the interconnection, and the damping matrix to get a desired Hamiltonian function or to improve the dynamic performance of the system.

Generally, for a dissipative Hamiltonian realization of the system in Equation (1), we have following result [4]:

Lemma 1 Consider the system in Equation (1). Assume there exist matrices $g^{\perp}(x), J_{d}(x)=-J_{d}^{T}(x)$, $R_{d}(x)=R_{d}^{T}(x) \geq 0$ and differentiable scalar function $H_{d}(x)$ such that the following partial differential equation (PDE) holds

$$
g^{\perp}(x) f(x)=g^{\perp}(x)\left[J_{d}(x)-R_{d}(x)\right] \nabla H_{d}(x)
$$

where $g^{\perp}$ is a left annihilator of $g(x)$, i.e., $g^{\perp}(x) g(x)=0$ and $H_{d}(x)$ is such that

$$
x_{*}=\arg \min H_{d}(x)
$$

with $x_{*}$ the desired equilibrium point to be stabilized. Then, under the following feedback controller

$$
u(x)=\left[g^{\perp}(x) g(x)\right]^{-1} g^{\perp}\left\{\left[J_{d}(x)-R_{d}(x)\right] \nabla H_{d}-f(x)\right\}
$$

the closed loop system can be represented as

$$
\dot{x}=\left[J_{d}-R_{d}(x)\right] \nabla H_{d}(x)
$$

with $x_{*}$ being a local stable equilibrium. Furthermore, if the largest invariant set

$$
\left\{\left.x \in R^{n}|| \nabla H_{d}(x)\right|^{T} R_{d}(x) \nabla H_{d}=0\right\}=\left\{x_{*}\right\}
$$

the closed loop system is asymptotically stable.

According to Lemma 1, the main procedure of the IDA-PBC methodology can be briefly described as follows:

(i) Fix the desired structure of the interconnection and damping matrix.

(ii) Derive a partial differential equation (PDE) parameterized by the chosen matrices whose solutions characterize all the energy functions that can be assigned.

(iii) Choose one solution that satisfies the minimum requirement and computer the control.

Thus, in order to formulate the considered nonlinear system within a PCH system, the key step is to find a solution to the PDE in Equation (3). In the next section, we construct the pon-parameterized IDA and parameterized IDA methods proposed in [4], in order to reassign the interconnection and dissipative matrices of the single machine infinite bus power system and formulate a feedback controller to improve its transient stability. 


\section{IDA-PBC Excitation Control of Power Systems}

Consider a single machine infinite bus power system as shown in Figure 1.

Figure 1. the configuration of a single machine infinite bus power system.

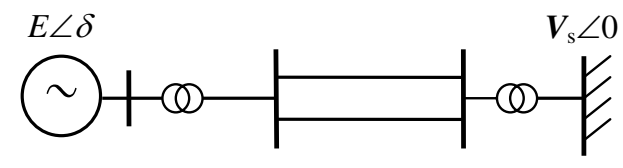

The dynamic of the system can be presented as

$$
\left\{\begin{array}{l}
\dot{\delta}=\omega-\omega_{0} \\
\dot{\omega}=\frac{\omega_{0}}{M} P_{m}-\frac{D}{M}\left(\omega-\omega_{0}\right)-\frac{\omega_{0} E_{q}^{\prime} V_{s} \sin \delta}{M x_{d \Sigma}^{\prime}} \\
\dot{E}_{q}^{\prime}=-\frac{E_{q}^{\prime}}{T_{d}^{\prime}}+\frac{x_{d}-x_{d}^{\prime}}{T_{d 0} x_{d \Sigma}^{\prime}} V_{s} \cos \delta+\frac{1}{T_{d 0}} V_{f}
\end{array}\right.
$$

where $\delta$ is the power angle of the generator, in radians $(\mathrm{rad}) ; \omega$ is the rotor speed of the generator, $\omega_{0}=2 \pi f_{0}$, in $\mathrm{rad} / \mathrm{s} ; E_{q}^{\prime}$ is the $q$-axis internal transient voltage of the generator, in per unit; $V_{f}$ is the voltage of the field circuit of the generator - the control input, in per unit; $P_{m}$ is the mechanical power, assumed to be constant, in per unit; $V_{s}$ is the voltage of the infinite bus, in per unit; $x_{d}^{\prime}$ is the $d$-axis transient reactance of the generator, in per unit; $x_{d}$ is the $d$-axis reactance, in per unit; $M$ is the inertia coefficient of the generator, in s; $D$ is the damping constant, in per unit; $T_{d 0}$ is the $d$-axis transient open-circuit time constant, in $\mathrm{s} ; T_{d}$ is the $d$-axis transient closed-circuit time constant, in $\mathrm{s}$; $x_{d \Sigma}^{\prime}=x_{d}^{\prime}+\frac{1}{2} x_{L}+x_{T}$, where $x_{L}$ is the reactance of the transmission line, in per unit; $x_{T}$ is the reactance of the transformers, in per unit.

Let $x_{1}=\delta, x_{2}=\omega-\omega_{0}, x_{3}=E_{q}^{\prime}$ and $u=V_{f}$. Denote $a=\frac{\omega_{0} P_{m}}{M}, b=\frac{D}{M}, c=\frac{\omega_{0} V_{s}}{M x_{d \Sigma}^{\prime}}, d=\frac{1}{T_{d}}$, and $e=\frac{x_{d}-x_{d}^{\prime}}{T_{d 0} x_{d \Sigma}^{\prime}} V_{s}$. Direct calculation shows that the system can be Hamiltonian realized as follows:

$$
\dot{x}=[J(x)-R(x)] \nabla H+g(x) u
$$

where $g(x)=\left[0,0, \frac{1}{T_{d 0}}\right]^{T}$. The interconnection and dissipative matrices are

$$
J(x)=\left[\begin{array}{ccc}
0 & 1 & 0 \\
-1 & 0 & 0 \\
0 & 0 & 0
\end{array}\right], \quad R(x)=\left[\begin{array}{ccc}
0 & 0 & 0 \\
0 & b & 0 \\
0 & 0 & \frac{e}{c}
\end{array}\right]
$$

respectively. The corresponding Hamiltonian function is

$$
H(x)=\frac{1}{2} x_{2}^{2}+\frac{c d}{2 e} x_{3}^{2}-c x_{3} \cos x_{1}-a x_{1}
$$

It can be verified that $H(x)$ possesses a strict local minimum at the equilibrium point $x_{1}^{*}=\frac{1}{2} \arcsin \left(\frac{2 a d}{c e}\right), x_{2}^{*}=0$, and $x_{3}^{*}=\frac{e}{d} \cos x_{1}^{*}$. 
Noticing that the largest invariant set $\{\dot{H}(x)=0\}$ contains only the equilibrium point, the power system can be stabilized by a damping injection controller as follows:

$$
u=-k L_{g} H(x)
$$

where $k>0$ is the feedback gain.

The closed loop system can be written as

$$
\dot{x}=\left[J(x)-R_{c}(x)\right] \nabla H
$$

where the dissipative matrix is

$$
R_{c}(x)=\left[\begin{array}{ccc}
0 & 0 & 0 \\
0 & b & 0 \\
0 & 0 & \frac{e}{c}+k
\end{array}\right]
$$

It can be seen from the matrix $R_{c}(x)$ that the damping injection excitation controller cannot change the mechanic-electric dynamic of the system and thus cannot effectively attenuate the oscillation.

In the following, we use the IDA-PBC method to design an effective excitation controller to improve the performance of the system. First, assume the desired dissipative Hamiltonian realization of the power system can be formulated as

$$
\dot{x}=\left(J_{d}(x)-R_{d}(x)\right) \nabla H_{d}
$$

where the dissipative matrix $R_{d}=R_{c}$. Choose the interconnection matrix as

$$
J_{d}(x)=\left[\begin{array}{ccc}
0 & 1 & \alpha \\
-1 & 0 & 0 \\
-\alpha & 0 & 0
\end{array}\right]
$$

Suppose the desired Hamiltonian function can be chosen as

$$
H_{d}(x)=H(x)+H_{i n j}(x)
$$

Let the feedback stabilization controller $u=\phi(x)$. Then the closed loop system satisfies

$$
\dot{x}=\left[\begin{array}{ccc}
0 & 1 & \alpha \\
-1 & -b & 0 \\
-\alpha & 0 & -\left(\frac{e}{c}+k\right)
\end{array}\right] \nabla\left(H(x)+H_{i n j}(x)\right)=\left[\begin{array}{ccc}
0 & 1 & 0 \\
-1 & -b & 0 \\
0 & 0 & -\frac{e}{c}
\end{array}\right] \nabla H(x)+\left[\begin{array}{c}
0 \\
0 \\
\frac{1}{T_{d 0}}
\end{array}\right] \phi(x)
$$

Thus we have

$$
\left\{\begin{array}{l}
\frac{\partial H_{i n j}}{\partial x_{3}}=0 \\
-\frac{\partial H}{\partial x_{1}}-b \frac{\partial H_{i n j}}{\partial x_{2}}=0 \\
u=\alpha\left(\frac{\partial H}{\partial x_{1}}+\frac{\partial H_{i n j}}{\partial x_{1}}\right)-k \frac{\partial H}{\partial x_{3}}
\end{array}\right.
$$


From above equations we can see that $H_{i n j}$ does not depend on $x_{3}$. Taking into consideration that

$$
\left\{\begin{array}{l}
\frac{\partial H}{\partial x_{1}}=c x_{3} \sin x_{1}-a \\
\frac{\partial H}{\partial x_{2}}=x_{2} \\
\frac{\partial H}{\partial x_{3}}=\frac{c d}{e} x_{3}-c \cos x_{1}
\end{array}\right.
$$

we have

$$
\begin{aligned}
& H_{i n j}=\psi\left(x_{1}\right)+\frac{1}{b}\left(a-c x_{3} \sin x_{1}\right) x_{2} \\
& u=\alpha\left(c x_{3} \sin x_{1}-a+\frac{\partial H_{i n j}}{\partial x_{1}}\right)-k\left(\frac{c d}{d} x_{3}-c \cos x_{1}\right)
\end{aligned}
$$

where $\psi\left(x_{1}\right)$ is chosen such that

$$
\left.\frac{\partial H_{d}}{\partial x}\right|_{\left(x_{1}^{*}, x_{2}^{*}, x_{3}^{*}\right)}=0,\left.\quad \operatorname{Hess}\left(H_{d}\right)\right|_{\left(x_{1}^{*}, x_{2}^{*}, x_{3}^{*}\right)}>0
$$

hold. Noticing that

$$
H_{d}=H+H_{i n j}=\frac{1}{2} x_{2}^{2}+\frac{c d}{2 e} x_{3}^{2}-c x_{3} \cos x_{1}-a x_{1}+\psi\left(x_{1}\right)+\frac{1}{b}\left(a-c x_{3} \sin x_{1}\right) x_{2}
$$

we have

$$
\left\{\begin{array}{l}
\frac{\partial H_{d}}{\partial x_{1}}=c x_{3} \sin x_{1}-a+\frac{\partial \psi}{\partial x_{1}}-\frac{c}{b} x_{2} x_{3} \cos x_{1} \\
\frac{\partial H_{d}}{\partial x_{2}}=x_{2}+\frac{1}{b}\left(a-c x_{3} \sin x_{1}\right) \\
\frac{\partial H_{d}}{\partial x_{3}}=\frac{c d}{e} x_{3}-c \cos x_{1}-\frac{c}{b} x_{2} \sin x_{1}
\end{array}\right.
$$

So, in order to guarantee that $H_{d}$ has a strict local minimum at the equilibrium point, we can choose

$$
\frac{\partial \psi\left(x_{1}\right)}{\partial x_{1}}=\text { const }=\gamma
$$

Let $\psi\left(x_{1}\right)=\gamma x_{1}$, then we have

$$
\operatorname{Hess}\left(H_{d}\right)=\left[\begin{array}{ccc}
c x_{3} \cos x_{1}+\gamma-\frac{c}{b} x_{2} x_{3} \sin x_{1} & \frac{c}{b} x_{3} \cos x_{1} & c \sin x_{1}+\frac{c}{b} \cos x_{1} \\
-\frac{c}{b} x_{3} \cos x_{1} & 1 & -\frac{c}{b} \sin x_{1} \\
c x_{1}-\frac{c}{b} x_{2} \cos x_{1} & -\frac{c}{b} \sin x_{1} & \frac{c d}{e}
\end{array}\right]
$$

and

$$
\operatorname{Hess}\left(H_{d}\left(x^{*}\right)\right)=\left[\begin{array}{ccc}
\frac{c e}{d} \cos ^{2} x_{1}^{*}+\gamma & \frac{c e}{b d} \cos ^{2} x_{1}^{*} & c \sin x_{1}^{*}+\frac{c}{b} \cos x_{1}^{*} \\
-\frac{c e}{b d} \cos ^{2} x_{1}^{*} & 1 & 1 \\
c \sin x_{1}^{*} & \frac{c}{b} \sin x_{1}^{*} & 1
\end{array}\right]
$$

In order to guarantee a positive definite $H_{d}\left(x^{*}\right)$, we must choose $\gamma$ such that

$$
\gamma>-\frac{c e}{d} \cos ^{2} x_{1}^{*} \quad \text { and } \quad \gamma>\frac{\eta}{\frac{c d}{e}-\frac{c^{2}}{b^{2}} \sin ^{2} x_{1}^{*}}
$$


hold simultaneously, where

$$
\eta=\frac{c^{3} e}{b^{3} d} \cos ^{3} x_{1}^{*} \sin x_{1}^{*}-c \sin ^{2} x_{1}^{*}-\frac{c}{b} \cos x_{1}^{*} \sin x_{1}^{*}-\frac{c^{3} e^{2}}{b^{3} d^{2}} \cos ^{4} x_{1}^{*} \sin x_{1}^{*}
$$

According to Lemma 1, under the desired Hamiltonian realization the stabilization control scheme of the system can be chosen as

$$
u=\alpha\left(c x_{3} \sin x_{1}-a+\gamma-\frac{c}{b} x_{2} x_{3} \cos x_{1}\right)-k\left(\frac{c d}{e} x_{3}-c \sin x_{1}\right)
$$

where $k>0$ is the feedback gain. The corresponding Hamiltonian function of the closed loop system is

$$
H_{d}=\frac{1}{2} x_{2}^{2}+\frac{c d}{2 e} x_{3}^{2}-c x_{3} \cos x_{1}+(\gamma-a) x_{1}+\frac{1}{b}\left(a-c x_{3} \sin x_{1}\right) x_{2}
$$

Remark 1 The internal transient voltage of the generator can be presented by measurable signals such as $P_{e}, Q_{e}$ and $V_{s}$. Noting that the active and reactive power of the generator can be written as $P_{e}=$ $\frac{E_{q}^{\prime} V_{s}}{x_{d \Sigma}^{\prime}} \sin \delta$ and $Q_{e}=\frac{E_{q}^{\prime} V_{s}}{x_{d \Sigma}^{\prime}} \cos \delta-\frac{V_{s}^{2}}{x_{d \Sigma}^{\prime}}$ respectively, we have $E_{q}^{\prime}=\frac{x_{d \Sigma}^{\prime}}{V_{s}^{2}} \sqrt{\left(Q_{e}-\frac{V_{s}^{2}}{x_{d \Sigma}^{\prime}}\right)^{2}+P_{e}^{2}}$.

\section{Simulation}

To show the effectiveness of the proposed controller, we compare it with the conventional IDA-PBC controller [14] and PSS+AVR controller [16]. During the simulation, the effects of generator fields and rotor circuit dynamics are included in the model of power system [16]. The simulation is completed on a professional power system simulation platform developed by the China Electric Power Research Institute.

Figure 2. Response of the rotor angle under a circuit fault.

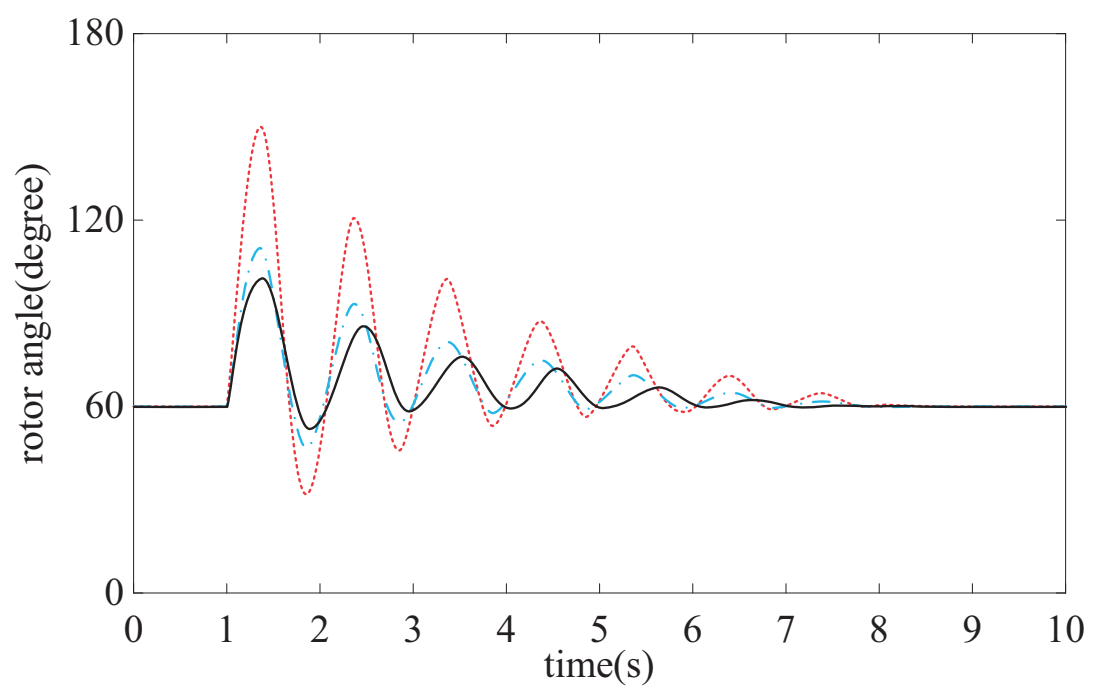

During the simulation, a three-phase temporary short-circuit fault is assumed to occur at the transmission line, starting at time $t=1 \mathrm{~s}$ and cleared at $t=1.1 \mathrm{~s}$. The parameters in the proposed feedback excitation controller are chosen as $k=2$ and $\gamma=5$. The simulation results are shown 
in Figures 2 and 3, where the solid black lines indicate the response under the proposed stabilization controller, the blue dashed lines corresponding to the response under conventional IDA-PBC controller and the red dotted lines correspond to PSS+AVR respectively.

Figure 3. Response of the terminal voltage of generator under a circuit fault.

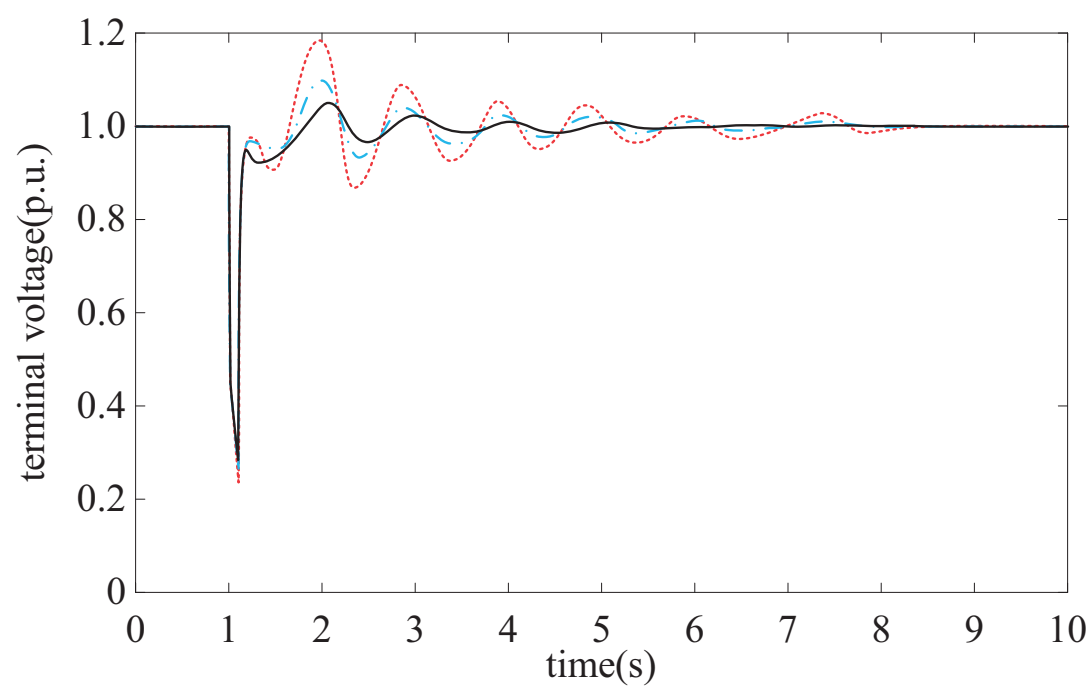

From the simulation results, it can be seen that, when the fault occurs, the proposed nonlinear stabilization control scheme makes the system responds much faster than the conventional passivity-based controller and PSS+AVR controller . It follows that the proposed controller outperforms the traditional controller in transient stability enhancement of the power system.

\section{Conclusions}

The excitation control of a single machine infinite bus power system based on the IDA-PBC method is investigated. We propose a novel excitation controller by choosing the desired interconnection and damping matrix, and solving the PDE to get the corresponding Hamiltonian function. An excitation controller is put forward based on the new Hamiltonian realization system, which not only injects damping into the electronic equation, but also reshapes the mechanical swing equation. Simulation results show that the proposed control scheme can effectively improve the transient stability of the power system.

\section{Acknowledgements}

This work was supported by the National Natural Science Foundation of China (No. 60974005 and 61104022), the Specialized Research Fund for the Doctoral Program of Higher Education (No. 20094101120008) and the Science and Technique Research Program of Henan Educational Committee (No. 13A520379).

\section{Conflict of Interest}

The authors declare no conflict of interest. 


\section{References}

1. Van der Schaft, A.J. Port-Hamiltonian Systems: Network Modeling and Control of Nonlinear Physical Systems. In Advanced Dynamics and Control of Structures and Machines; CISM Courses and Lectures, Volume 444; Springer: New York, NY, USA, 2004.

2. Ortega, R.; van der Schaft, A.J.; Mareels, I.; Maschke, B. Putting energy back in control. IEEE Control Syst. Mag. 2001, 21, 18-33.

3. Ortega, R.; van der Schaft, A.J.; Maschke, B.; Escobar, G. Interconnection and damping assignment passivity-based control of port-controlled Hamiltonian systems. Automatica 2002, 38, 585-596.

4. Ortega, R.; García-Causeco, E. Interconnection and damping assignment passivity-based control: A survey. Eur. J. Control 2004, 10, 432-450.

5. Ortega, R.; Castaños, F.; Astolfi, A. Control by interconnection and standard passivity-based control of Port-Hamiltonian systems. IEEE Trans. Autom. Control 2008, 53, 2527-2542.

6. Fujimoto, K.; Sakurama, K.; Surgie, T. Trajectory Tracking Control of Portcontrolled Hamiltonian Systems and its Application to a Magnetic Levitation System. In Proceedings of the 40th IEEE Conference on Decision and Control, Orlando, FL, USA, 4-7 December 2001; pp. 3388-3393.

7. Rodríguez, H.; Ortega, R. Stabilization of electromechanical systems via interconnection and damping assignment. Int. J. Robust Nonlinear Control 2003, 13, 1095-1111.

8. Batlle, C.; Dòria-Cerezo, A.; Fossas, E. Bidirectional power flow control of a power converter using passive Hamiltonian techniques. Int. J. Circuit Theory Appl., 2008, 36, 769-788.

9. Cheng, D.; Xi, Z.; Hong, Y.; Qin, H. Energy-based Stabilization in Power Systems. In Proceedings of the 14th IFAC World Congress, Beijing, China, 5-9 July 1999; pp. 297-303.

10. Xi, Z.; Cheng, D.; Lu, Q.; Mei, S. Nonlinear decentralized controller design for multimachine power systems using Hamiltonian function method. Automatica 2002, 38, 527-534.

11. Wang, Y.; Cheng, D.; Hong, Y. Stabilization of synchronous generators with Hamiltonian function approach. Int. J. Syst. Sci. 2001, 32, 971-978.

12. Sun, Y.; Shen, T.; Ortega, R.; Lu, Q. Decentralized Controller Design for Multi-machine Power Systems on Hamiltonian Structure. In Proceedings of the 40th IEEE Conference on Decision and Control, Orlando, USA, 4-7 December 2001; pp. 3045-3050.

13. Xi, Z.; Cheng, D.; Lu, Q.; Mei, S. Nonlinear decentralized controller design for multimachine power systems using Hamiltonian function method. Automatica 2002, 38, 527-534.

14. Galaz, M. ; Ortega, R. ; Bazanella, A.S.; Stankovic, A.M. An energy-shapping approach to the design of excitation control of synchronous generators. Automatica 2003, 39, 111-119.

15. Li, J.; Liu, Y.; Li, C.; Zhang, L. Interconnection and Damping Assignment Passivity-based Excitation Control of Power Systems. In Proceedings of IEEE Control and Decision Conference, Xuzhou, China, 26-28 May 2010; pp. 954-958.

16. Kundur, P. Power System Stability and Control; McGraw-Hill: New York, NY, USA, 1994.

(C) 2013 by the authors; licensee MDPI, Basel, Switzerland. This article is an open access article distributed under the terms and conditions of the Creative Commons Attribution license (http://creativecommons.org/licenses/by/3.0/). 